\title{
The split Renilla luciferase complementation assay is useful for identifying the interaction of Epstein-Barr virus protein kinase BGLF4 and a heat shock protein Hsp90
}

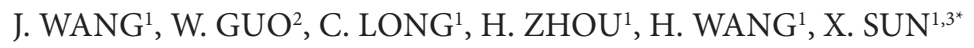

\begin{abstract}
${ }^{1}$ Department of Pathogen Biology, School of Basic Medical Sciences, Wuhan University, Wuhan, 430071, P. R. China; ${ }^{2}$ Department of Pathology and Physiology, School of Basic Medical Sciences, Wuhan University, Wuhan, 430071, P. R. China; ${ }^{3}$ State Key laboratory of Virology, Wuhan University, Wuhan, 430072, P. R. China
\end{abstract}

Received May 21, 2015; revised July 14, 2015; accepted February 15, 2016

\begin{abstract}
Summary. - Protein-protein interactions can regulate different cellular processes, such as transcription, translation, and oncogenic transformation. The split Renilla luciferase complementation assay (SRLCA) is one of the techniques that detect protein-protein interactions. The SRLCA is based on the complementation of the LN and LC non-functional halves of Renilla luciferase fused to possibly interacting proteins which after interaction form a functional enzyme and emit luminescence. The BGLF4 of Epstein-Barr virus (EBV) is a viral protein kinase that is expressed during the early and late stages of lytic cycles, which can regulate multiple cellular and viral substrates to optimize the DNA replication environment. The heat shock protein Hsp90 is a molecular chaperone that maintains the integrity of structure and function of various interacting proteins, which can form a complex with BGLF4 and stabilize its expression in cells. The interaction between BGLF4 and Hsp90 could be specifically detected through the SRLCA. The region of aa 250-295 of BGLF4 is essential for the BGLF4/Hsp90 interaction and the mutation of Phe-254, Leu-266, and Leu-267 can disrupt this interaction. These results suggest that the SRLCA can specifically detect the BGLF4/Hsp90 interaction and provide a reference to develop inhibitors that disrupt the BGLF4/Hsp90 interaction.
\end{abstract}

Keywords: split Renilla luciferase complementation assay; protein-protein interaction; BGLF4; Hsp90

\section{Introduction}

Protein-protein interactions play an important role in regulating different cellular processes, including the transcription, translation, and oncogenic transformation. Several techniques have been developed to study biological interactions between proteins in cells, especially the split Renilla luciferase

*Corresponding author. E-mail: xsun6@whu.edu.cn; phone: +862768759361.

Abbreviations: BGLF4 = Epstein-Barr virus protein kinase; $\mathrm{EBV}=$ Epstein-Barr virus; Hsp90 = $90 \mathrm{~K}$ heat shock protein; $\mathrm{LC}=\mathrm{C}$-terminal domains of Renilla luciferase; $\mathrm{LN}=\mathrm{N}$-terminal domains of Renilla luciferase; SRLCA = split Reinlla luciferase complementation assay complementation assay (SRLCA) (Hattori and Ozawa, 2014; Paulmurugan and Gambhir, 2003; Rossi et al., 1997). The Renilla luciferase is an ideal reporter in mammalian cells, because its activity is independent of ATP or post-translational modification. The $\mathrm{N}$-terminal fragment consisting of aa 1-229 and the C-terminal fragment consisting of aa 230-311 of the $36 \mathrm{~K}$ Renilla luciferase were shown to have the most efficient complementation of luciferase activity (Paulmurugan and Gambhir, 2003). The SRLCA is based on the principle that the $\mathrm{N}$ and $\mathrm{C}$ termini of Renilla luciferase alone do not radiate luminescence; however, if fused with interacting proteins the non-functional parts will be joined together to form a functional enzyme and emit luminescence in the presence of the substrate coelenterazine. Therefore, the specific proteinprotein interaction can be detected by measuring Renilla luciferase activity in the microplate luminometer. 
Epstein-Barr virus (EBV) is a ubiquitous gammaherpesvirus, which infects most of the human population and persists in the host for life. EBV is closely associated with various human malignant diseases, including Burkitt and Hodgkin lymphoma, nasopharyngeal carcinoma, gastric carcinoma, and lymphoproliferative disease. EBV protein kinase BGLF4 (encoded by the BGLF4 gene) is a serine/threonine protein kinase that is expressed during the early and late stages of lytic cycles (Wang et al., 2005). As a member of the conserved herpesviral protein kinases, BGLF4 has been identified in all human herpesviruses (Chee et al., 1989). A number of viral cellular proteins, the latent viral proteins EBNA1 (Zhu et al., 2009), the DNA polymerase processivity factor BMRF1 (Yang et al., 2008), the EBV immediate early protein BZLF1 (Asai et al., 2006), the interferon regulatory factor 3 (IRF3) (Wang et al., 2009), the cell cycle regulatory protein p27 (Iwahori et al., 2009), and nuclear lamin A/C (Lee et al., 2008) are phosphorylated by BGLF4. BGLF4 also modulates the structure and transport preference of nuclear pore complex to facilitate nuclear import of EBV lytic proteins (Chang et al., 2015).

Heat shock proteins are a group of conserved molecular chaperones that facilitate proper protein folding, intracellular trafficking, stability, and interactions (Jego et al., 2013; Nahleh et al., 2012; Whitesell and Lindquist, 2005), which are expressed by the cell in response to stress conditions. As an evolutionarily conserved molecular chaperone, Hsp90 plays an essential role in many cellular processes, including cell survival, transcriptional regulation, and signal transduction (Pearl and Prodromou, 2000). Interacting proteins of Hsp90 include steroid hormone receptors, transcription factors, protein kinases, and enzymes. Interestingly, cellular protein kinases account for a large part of Hsp90 interacting proteins; indeed, more than half of the known human protein kinases were shown to interact with Hsp90 (Taipale et al., 2012).

We have previously demonstrated that BGLF4 associates with Hsp90 (Sun et al., 2013). However, the region of BGLF4 that is important for BGLF4/Hsp90 interaction was not defined yet. Consistent with previous study, the data presented here show that BGLF4 has a strong interaction with Hsp90. By analyzing deletion mutants and point mutations of BGLF4, the data show that the aa 250-295 of BGLF4 play a crucial role in the interaction of BGLF4/Hsp90. Furthermore, Phe-254, Leu-266, and Leu-267 amino acid residues of BGLF4 are important for the BGLF4/Hsp90 interaction and the mutation of these amino acids can disrupt the interaction between BGLF4 and Hsp90. These results provide a reference to develop inhibitors that would disrupt the interaction between BGLF4 and Hsp90.

\section{Materials and Methods}

Cell lines and transfection. Human renal embryonic 293T cells were kindly provided by Prof. Z. Yang (Wuhan University,
China). EBV-positive cell line B95-8 was obtained from Prof. Y. Cao (Central South University, China). 293T cells were cultured in Dulbecco's modified Eagle's medium (DMEM) (Hyclone, USA) containing 10\% FBS (Gibco, USA) and B95-8 cells were grown in RPMI-1640 media (Hyclone, USA) containing $10 \% \mathrm{FBS}$ at $37^{\circ} \mathrm{C}$ with $5 \% \mathrm{CO}_{2}$. Plasmids were transiently transfected into $293 \mathrm{~T}$ cells using X-tremeGENE HP DNA transfection reagent (ROCHE, Switzerland) according to the manufacturer's instructions.

Plasmids. The plasmids LN and LC were kindly provided by Prof. F. Li (South Dakota State University, South Dakota State, USA). The plasmids pcDNA3.1-BGLF4 and pcDNA3.1-Hsp90 were constructed by inserting the full-length BGLF4 and Hsp90 sequences into pcDNA3.1 plasmid. The corresponding fragments were amplified from the cDNA of BGLF4 and Hsp90 using primers BGLF4 NotI-F/BGLF4 XbaI-R and Hsp90 NotI-F/Hsp90 XbaI-R. The cDNA was synthesized by reverse transcriptase from the total RNA extracted from B95-8 cells. The BGLF4 NotI/XbaI and Hsp90 NotI/XbaI restriction sites present in the primers were adjusted by digestion and inserted into the NotI/XbaI sites of pcDNA3.1 plasmids (YouBio, China). All primers used in this study are listed in Table 1. The HA-tagged BGLF4 was amplified from pcDNA3.1BGLF4 using primers BGLF4 XbaI HA-F and BGLF4 EcoRI-R, and subcloned upstream of LC or LN with a linker (G4S)2 using the $\mathrm{XbaI}$ and EcoRI restriction enzymes (NEB, USA). Another HA-tagged BGLF4 was amplified from pcDNA3.1-BGLF4 using primers BGLF4 EcoRI-F and BGLF4 NotI HA-R, and subcloned downstream of LC or LN with a linker (G4S)2 using the EcoRI and NotI (NEB, USA). The Hsp90 was amplified from pcDNA3.1Hsp90 using primers Hsp90 XhoI-F and Hsp90 ApaI-R, and subcloned upstream of LC or LN through a linker (G4S)2 using the XhoI and ApaI (NEB, USA). Another Hsp90 was amplified from pcDNA3.1-Hsp90 using primers Hsp90 XhoI-F and Hsp90 NotI-R, and subcloned downstream of LC or LN through a linker (G4S)2 using the XhoI and NotI restriction enzymes. All mutants with deletion and point mutations of BGLF4 tagged by HA and linked to LN were generated by the PCR. BGLF4 d(1-160) was amplified from pcDNA3.1-BGLF4 using primers BGLF4 $160 \mathrm{XbaI}$ HA-F and BGLF4 EcoRI-R. After amplification, BGLF4 d(1-160) was digested with $\mathrm{XbaI} / E c o R I$ and ligated into the $\mathrm{XbaI} / E c o R I$ sites of LN plasmid to construct the BGLF4 d(1-160)-LN. BGLF4 $\mathrm{d}(282-428)$ was amplified from pcDNA3.1-BGLF4 using primers BGLF4 XbaI HA-F and BGLF4 282 EcoRI-R, and cloned into the $\mathrm{XbaI} / E c o R I$ sites of LN plasmid to construct the BGLF4 d(282-428)-LN. The forward primer for BGLF4 (1-146), BGLF4 (1-150), BGLF4 (1-175), BGLF4 (1-215), and BGLF4 (1-250) is BGLF4 Xbal HA-F, and the reverse primers are as follows: BGLF4 146-R, BGLF4 150-R, BGLF4 175-R, BGLF4 215-R, and BGLF4 250-R. The reverse primer for BGLF4 (180-428), BGLF4 (220-428), BGLF4 (254-428), BGLF4 (293-428), and BGLF4 (295-428) is BGLF4 EcoRI-R, and the forward primers are as follows: BGLF4 180-F, BGLF4 220-F, BGLF4 254-F, BGLF4 293-F, and BGLF4 295-F. Pairs of products that were amplified from pcDNA3.1-BGLF4, BGLF4 (1-146)/BGLF4 (180-428), BGLF4 (1-175)/BGLF4 (220-428), 
Table 1. PCR primers used in this study

\begin{tabular}{|c|c|}
\hline Primer name & Primer sequence $\left(5^{\prime} \rightarrow 3^{\prime}\right)$ \\
\hline BGLF4 EcoRI-F & ATCGGAATTCATGGATGTGAATATGGCTGCG \\
\hline BGLF4 NotI-F & CTAGGCGGCCGCATGGATGTGAATATGGC \\
\hline BGLF4 XbaI-F & CTAGTCTAGAGATGGATGTGAATATGGCTG \\
\hline Hsp90 NotI-F & CTAGGCGGCCGCATGCCTGAGGAAACCCAG \\
\hline Hsp90 XhoI-F & ATCGCTCGAGATGCCTGAGGAAACCCAG \\
\hline BGLF4 XbaI HA-F & AGTCTAGAGATGTACCCATACGATGTTCCAGATTACGCTGATGTGAATATGGC \\
\hline BGLF4 160 XbaI HA-F & TAGTCTAGAGATGTACCCATACGATGTTCCAGATTACGCTTATGGCCACTGGCATG \\
\hline BGLF4 180-F & GCCTGCACGTCCTGCGCCGTTTACTTTCTG \\
\hline BGLF4 220-F & CTGGTGCGGGGCGGGACTGCTTCCCTC \\
\hline BGLF4 254-F & GCATGGGTAGGTCTATAGCCAAGGAC \\
\hline BGLF4 293-F & CGCCTCTATTGCCTGGATCTGCAGTCG \\
\hline BGLF4 295-F & CGCCTCTATTGCCTGGATCTGCAGTCG \\
\hline BGLF4 (Q250A)-F & CGCCTCTATTGCGCTAGGGAACCATTTTCTATAGC \\
\hline BGLF4 (F254A)-F & TGCCAGAGGGAACCAGCTTCTATAGCCAAGGAC \\
\hline BGLF4 (L266A)-F & AAGCCCCTCTGCGCTTTGAGCAAGTGCTACATC \\
\hline BGLF4 (L267A)-F & AAGCCCСTCTGCCTTGCTAGCAAGTGCTACATC \\
\hline BGLF4 (R274A)-F & AAGTGCTACATCTTGGCTGGGGCTGGGCACATC \\
\hline BGLF4 (L266A/L267A)-F & AAGCCCCTCTGCGCTGCTAGCAAGTGCTACATC \\
\hline BGLF4 EcoRI-R & GACGGAATTCGCTCCACGTCGGCCATCTGG \\
\hline BGLF4 NotI HA-R & CGATGCGGCCGCTTAAGCGTAATCTGGAACATCGTA TGGGTATCCACGTCGGC \\
\hline BGLF4 XbaI-R & GCATTCTAGATCATCCACGTCGGCCATCTG \\
\hline Hsp90 ApaI-R & CTGAGGGCCCGTCTACTTCTTCCATGCGTG \\
\hline Hsp90 XbaI-R & GATCTCTAGATTAGTCTACTTCTTCCATGCG \\
\hline Hsp90 NotI-R & CGATGCGGCCGCTTAGTCTACTTCTTCCATGCG \\
\hline BGLF4 282 EcoRI-R & GACGGAATTCGCGGGGTCAGGGATGTGCCCAG \\
\hline BGLF4 146-R & CAGAAAGTAAACGGCGCAGGACGTGCAGGC \\
\hline BGLF4 150-R & CAGATCCAGGCGAAGGGCCATAAACAGGGCGTG \\
\hline BGLF4 175-R & GAGGGAAGCAGTCCCGCCCCGCACCAG \\
\hline BGLF4 215-R & GTCCTTGGCTATAGACCTACCCATGC \\
\hline BGLF4 250-R & CGACTGCAGATCCAGGCAATAGAGGCG \\
\hline BGLF4 (Q250A)-R & GCGGAGATAACGCGATCCCTTGGTAAAAGATATCG \\
\hline BGLF4 (F254A)-R & ACGGTCTCCCTTGGTCGAAGATATCGGTTCCTG \\
\hline BGLF4 (L266A)-R & TTCGGGGAGACGCGAAACTCGTTCACGATGTAG \\
\hline BGLF4 (L267A)-R & TTCGGGGAGACGGAACGATCGTTCACGATGTAG \\
\hline BGLF4 (R274A)-R & TTCACGATGTAGAACCGACCCCGACCCGTGTAG \\
\hline BGLF4 (L266A/L267A)-R & TTCGGGGAGACGCGACGATCGTTCACGATGTAG \\
\hline
\end{tabular}

BGLF4 (1-215)/BGLF4 (254-428), BGLF4 (1-150)/BGLF4 (293-428), and BGLF4 (1-250)/BGLF4 (295-428) were respectively combined and amplified with primers BGLF4 XbaI HA-F and BGLF4 EcoRI-R to form the final PCR products BGLF4 d(146-180), BGLF4 d(175-220), BGLF4 d(215-254), BGLF4 d(150-293), and BGLF4 d(250-295). The final PCR products were digested and ligated into the $\mathrm{XbaI} / \mathrm{EcoRI}$ sites of $\mathrm{LN}$ plasmid to form deletion mutants BGLF4 d(146-180)-LN, BGLF4 d(175-220)-LN, BGLF4 d(215-254)-LN, BGLF4 d(150-293)-LN, and BGLF4 d(250-295)LN. The point mutants BGLF4 (Q250A)-LN, BGLF4 (F254A)-LN, BGLF4 (L266A)-LN, BGLF4 (L267A)-LN, BGLF4 (R274A)-LN, and BGLF4 (L266A/L267A)-LN were amplified from BGLF4-LN using the pairs of primers BGLF4 (Q250A)-F/BGLF4 (Q250A)-R, BGLF4 (F254A)-F/BGLF4 (F254A)-R, BGLF4 (L266A)-F/BGLF4 (L266A)-R, BGLF4 (L267A)-F/BGLF4 (L266A)-R, BGLF4 (R274A)-F/BGLF4 (R274A)-R, and BGLF4 (L266A/L267A)-F/ BGLF4 (L266A/L267A)-R, respectively. The different mutations were confirmed by DNA sequencing. All plasmids were purified through a purification kit (Axygen, USA) as described by the manufacturer.

Western blot analysis. 293T cells were seeded in to 6-well plates and transiently transfected with constructed plasmids. At $48 \mathrm{hr}$ post 
(a)

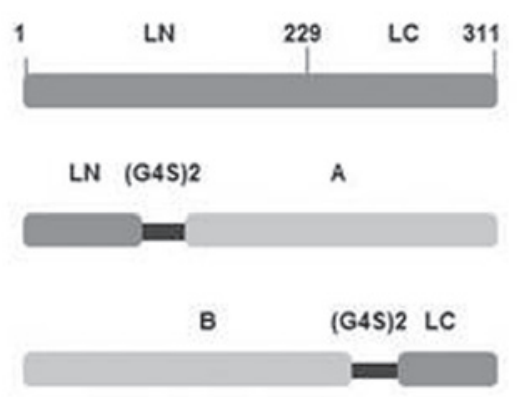

(b)

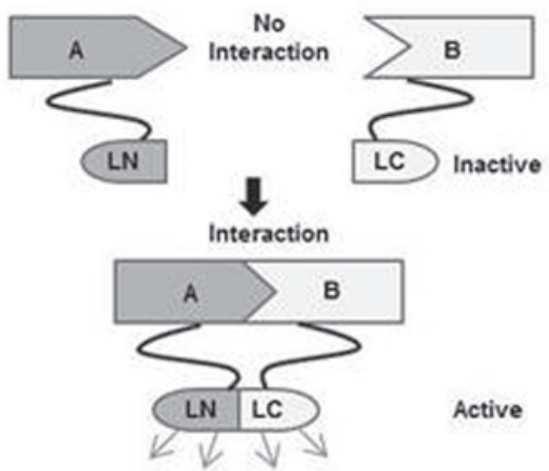

Fig. 1

The strategy of split Renilla luciferase complementation assay for detection of protein-protein interactions (a) Proteins A and B were fused to the LN (aa 1-229) and LC (aa 230-311) fragments of Renilla luciferase through the linker peptide (G4S)2. (b) LN and LC halves of Renilla luciferase remain non-functional in the absence of an interaction between A and B proteins. In contrast, the interaction of A and B can bring the LN and LC together and recover luciferase activity and exhibit emission of light in the presence of the substrate coelenterazine. Data are presented as mean \pm S.D. $(n=6)$.

transfection, cells were washed twice with cold phosphate buffered saline (PBS) and collected in RIPA lysis buffer (Beyotime, China). After centrifugation at 13,000 $\mathrm{x}$ g for $15 \mathrm{~min}$, the supernatant was transferred into new tubes, mixed with $5 \mathrm{x}$ loading buffer $(50 \%$ glycerol, 10\% SDS, 5\% $\beta$-mercaptoethanol, $0.5 \% \mathrm{BPB}, 250 \mathrm{nmol} / \mathrm{l}$ Tris- $\mathrm{HCl}(\mathrm{pH} 6.8)$ ) and boiled for $5 \mathrm{~min}$ at $95^{\circ} \mathrm{C}$. The total proteins were separated on $10 \%$ SDS-polyacrylamide gel and subjected to western blot analyses. The primary antibodies used were: Hsp90 (1:1,000; Abcam, UK), HA (1:10,000; Proteintech, China), and GAPDH (1:50,000; Proteintech). The secondary antibodies used were horseradish-peroxidase-conjugated secondary anti-mouse $\operatorname{IgG}(1: 10,000$; Kerui tech, China) and anti-rabbit $\operatorname{IgG}(1: 10,000$; Kerui tech). The proteins were detected by the ECL system (BIO RAD, USA).

Immunoprecipitation assay. 293T cells were seeded in to $100 \mathrm{~mm}$ tissue culture dishes and transfected with constructed plasmids. After $48 \mathrm{hr}$, cells were washed with cold PBS and collected in RIPA buffer. After centrifugation at 13,000 x g for $15 \mathrm{~min}$, supernatant was first incubated with $\mathrm{Hsp} 90$ antibody at $4{ }^{\circ} \mathrm{C}$ for $2 \mathrm{hr}$, followed by addition of $20 \mu \mathrm{l}$ of protein $\mathrm{A}+\mathrm{G}$-agarose (Beyotime, Shanghai, China) and incubation at $4{ }^{\circ} \mathrm{C}$ overnight. Beads were collected and washed thrice with cold PBS. Precipitates were resuspended with lysis buffer and separated on 8\% SDS-PAGE gel and subjected to western blot analyses.

Split Renilla luciferase complementation assay. Protein-protein interactions can be detected by SRLCA. In the assay, proteins $A$ and B were fused to the LN (aa 1-229) and LC (aa 230-311) fragments of Renilla luciferase through the linker peptide (G4S)2 (Fig. 1a). The SRLCA is based on the principle that Renilla luciferase halves (LN and LC) remain non-functional in the absence of an interaction between proteins $\mathrm{A}$ and $\mathrm{B}$. In contrast, the interaction between $\mathrm{A}$ and $\mathrm{B}$ can bring the $\mathrm{LN}$ and $\mathrm{LC}$ together and recover luciferase activity and exhibit emission of light in the presence of the substrate coelenterazine (Fig. 1b). 293T cells were seeded in to 12 -well plates and transfected with indicated pairs of fusion plasmids ( $0.5 \mu \mathrm{g}$ each plasmid). After $48 \mathrm{hr}$, cells were washed twice with cold PBS and lysed with passive lysis buffer to determine luciferase activity as a function of BGLF4/Hsp90 interactions using the Renilla luciferase assay system (Promega, USA) and measured by the GloMax 20/20 luminometer (Promega) according to the manufacture's recommendations.

Statistical analysis. Data were statistically evaluated using GraphPad Prism (GraphPad Software, USA) and shown as the mean \pm standard deviation.

\section{Results}

\section{SRLCA detects the BGLF4/Hsp90 interaction}

To detect the interaction between BGLF4 and Hsp90, fusion constructs representing the BGLF4 and Hsp90 proteins were constructed in multiple configurations to avoid the wrong orientation of fusion protein, which may sterically affect the association of LC and LN fragments subsequently repressing the activity of Renilla luciferase (Fig. 2a). All possible combinations of SRLCA BGLF4/Hsp90 pairs were transfected into $293 \mathrm{~T}$ cells for $48 \mathrm{hr}$ and detected by the luciferase activity in the luciferase assay system. As shown in Fig. 2b, all pairs exhibited luciferase activity, especially the pair of BGLF4-LN/LC-Hsp90 that has the highest luciferase activity ( 13,500 RLU). These results suggest that SRLCA can detect the interaction of BGLF4/Hsp90 and the pair BGLF4LN/LC-Hsp90 has the most powerful interaction. Therefore, 
(a)

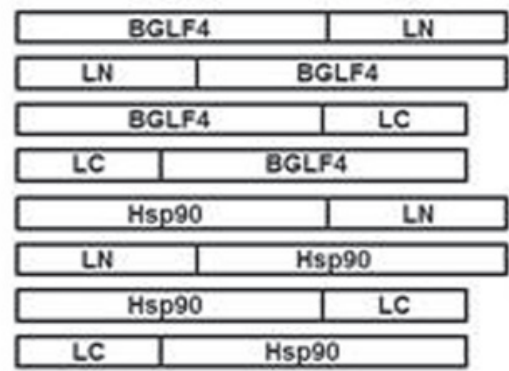

(c)

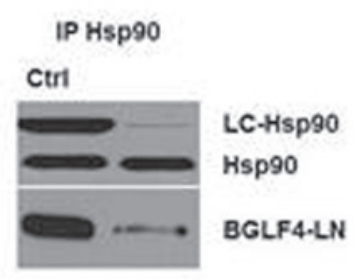

(b)

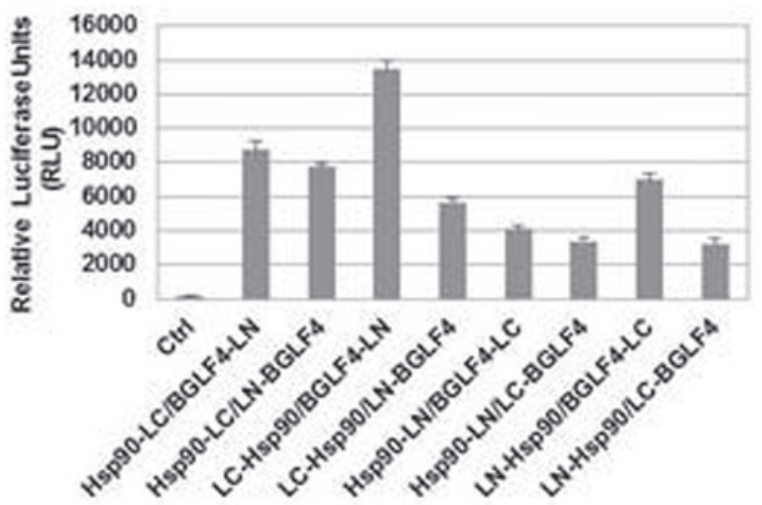

(d)

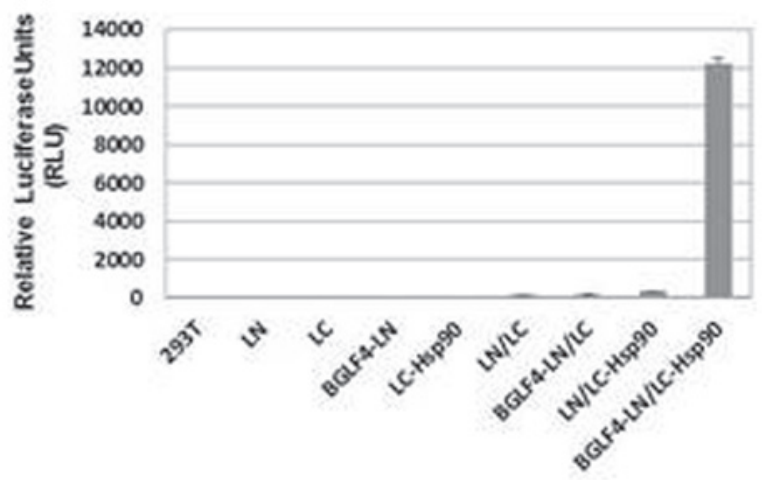

Fig. 2

SRLCA is specific for detecting the BGLF4/Hsp90 interaction

(a) Proteins BGLF4 and Hsp90 were fused through the linker (G4S)2 to the C-terminus or N-terminus of LC or LN fragments of Renilla in different conformations. (b) The luciferase assay of 293T cells transfected with different pairs of plasmids, after $48 \mathrm{hr}$. (c) Immunoprecipitation of $293 \mathrm{~T}$ cells cotransfected with BGLF4-LN and LC-Hsp90 for $48 \mathrm{hr}$, with antibody against Hsp90. Cell lysates without immunoprecipitation were used as control. Western blot analyses was performed with Hsp90 and HA antibodies. (d) The luciferase assay of 293T cells transfected with LN, LC, BGLF4-LN, LC-Hsp90, LN/ LC, BGLF4-LN/LC, LN/LC-Hsp90, and BGLF4-LN/LC-Hsp90, after 48 hr. Data are presented as mean \pm S.D. $(n=6)$.

the BGLF4-LN and LC-Hsp90 pair was selected to study the BGLF4/Hsp90 interaction through the SRLCA.

To test if fusion proteins BGLF4-LN and LC-Hsp90 could interact with each other in cells, immunoprecipitation was performed after BGLF4-LN and LC-Hsp90 plasmids were co-transfected into 293T cells. As shown in Fig. 2c, Hsp90 and fusion protein LC-Hsp90 were precipitated by Hsp90 antibody. The immunoprecipitated complex was also probed with anti-HA antibody. These results suggest that fusion proteins BGLF4-LN and LC-Hsp90 preserve the function of BGLF4 and Hsp90 and can interact with each other in cells.

To test the specificity of SRLCA, 293T cells were transfected with LN, LC, LC-Hsp90, BGLF4-LN, LN/LC, LN/ LC-Hsp90, LC/BGLF4-LN, and LC-Hsp90/BGLF4-LN, respectively. Forty-eight hours after transfection, cell lysates were collected to measure the luciferase activity. As shown in Fig. 2d, the luciferase activity did not increase observably when $293 \mathrm{~T}$ cells were transfected with LN, LC, LC-Hsp90, BGLF4-LN, LN/LC, LN/LC-Hsp90, and LC/BGLF4-LN. However, the luciferase activity of BGLF4-LN/LC-Hsp90 was enhanced by 80.17 -folds, 73.29-folds, or 35.89-folds compared with the transfection of LN/LC, LC/BGLF4-LN, and LN/LC-Hsp90. These results suggest that the SRLCA method is specific to detect the interaction of BGLF4/Hsp90.

The aa 250-295 of BGLF4 is essential for the interaction between BGLF4 and Hsp90

To investigate if the functional domains of BGLF4 contribute to its interaction with Hsp90, deletion mutants d(1-160) (deletion of aa 1-160), d(150-293), and d(282-428) of BGLF4 were tagged by HA and linked to the LN terminal of Renilla luciferase through the linker (G4S)2 (Fig. 3a). These deletion mutants were transfected into 293T cells and cultivated for $48 \mathrm{hr}$. Cells were collected to detect the expressed 
(a)

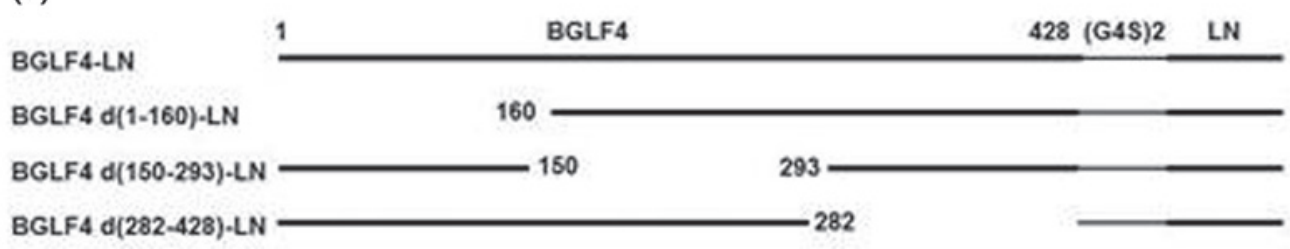

(b)

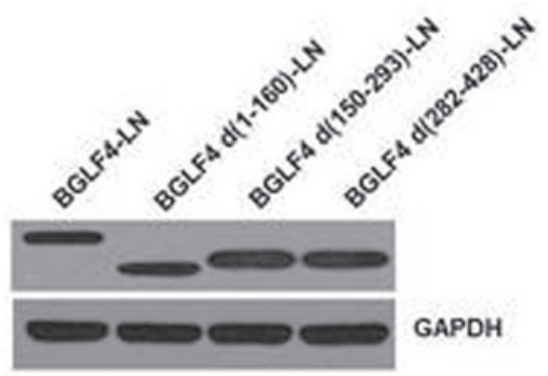

(c)

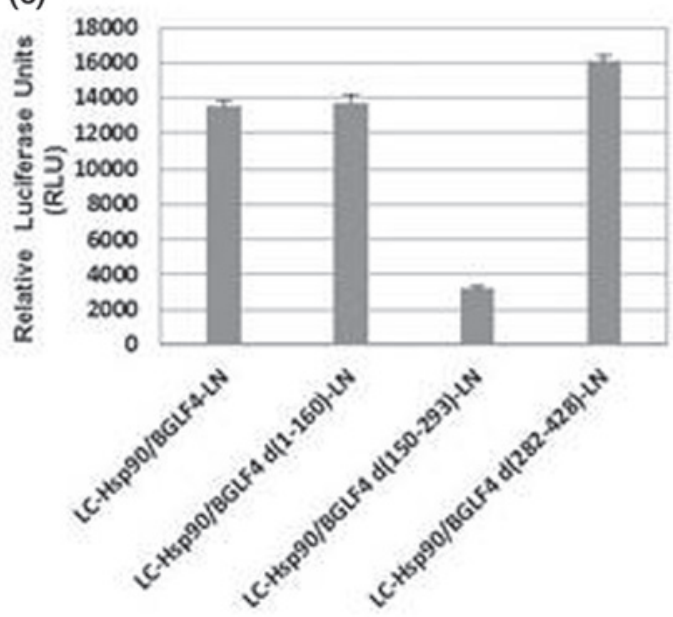

Fig. 3

Luciferase assay for BLGF4 deletion mutants

(a) The deletion mutants $\mathrm{d}(1-160), \mathrm{d}(150-293)$, and $\mathrm{d}(282-428)$ of BGLF4 tagged by HA and linked to the LN via the linker (G4S)2. (b) Western blot analysis of 293T cells transfected with deletion mutants BGLF4 d(1-160)-LN, BGLF4 d(150-293)-LN, and BGLF4 d(282-428)-LN after $48 \mathrm{hr}$, using the antibody against HA. (c) Luciferase assay of 293T cells transfected with deletion mutants BGLF4 d(1-160)-LN, BGLF4 d(150-293)-LN, and BGLF4 $\mathrm{d}(282-428)-\mathrm{LN}$ and LC-Hsp90 after $48 \mathrm{hr}$. Data are presented as mean \pm S.D. $(\mathrm{n}=6)$.

fusion protein using western blot analysis. As demonstrated in Fig. 3b, the specific bands of recombinant BGLF4 d(1-160)-LN, BGLF4 d(150-293)-LN, and BGLF4 d(282-428)-LN fusion proteins could be detected in transfected cells. To test the interaction of BGLF4 d(1-160)-LN, BGLF4 d(150-293)-LN, and BGLF4 d(282-428)-LN with LC-Hsp90, deletion mutants were co-transfected with LC-Hsp90 into 293T cells. Cells were collected to measure the luciferase activity using the Renilla luciferase assay system. As shown in Fig. 3c, the luciferase activity of the pair of BGLF4 d(150-293)-LN/ LC-Hsp90 decreased by about 70\% compared with the pair of full-length BGLF4-LN/LC-Hsp90. These results suggest that the aa 150-293 of BGLF4 are essential for the BGLF4/ Hsp90 interaction.

In order to determine if the specific aa 150-293 of BGLF4 interact with Hsp90, the deletion mutants d(146-180), $\mathrm{d}(175-220), \mathrm{d}(215-254)$, and $\mathrm{d}(250-295)$ of BGLF4 were tagged by $\mathrm{HA}$ and linked to the $\mathrm{LN}$ through the linker (G4S)2 (Fig. 4a). Deletion mutants BGLF4 d(146-180)-LN, BGLF4 d(175-220)-LN, BGLF4 d(215-254)-LN, and BGLF4 $\mathrm{d}(250-295)-\mathrm{LN}$ were transfected into $293 \mathrm{~T}$ cells and the expression was proved by using western blot analyses (Fig.4b).
To test the luciferase activity of BGLF4 d(146-180)-LN, BGLF4 d(175-220)-LN, BGLF4 d(215-254)-LN, and BGLF4 d(250-295)-LN with LC-Hsp90, these deletion mutants were co-transfected with LC-Hsp90 into 293T cells. After 48 $\mathrm{hr}$, the luciferase activity was detected by using the Renilla luciferase assay system. As shown in Fig. 4c, the luciferase activity did not change obviously when $293 \mathrm{~T}$ cells were transfected with the pairs of BGLF4 d(146-180)-LN/LC-Hsp90, BGLF4 d(175-220)-LN/LC-Hsp90, and BGLF4 d(215-254)LN/LC-Hsp90. However, the luciferase activity of the pair of BGLF4 d(250-295)-LN/LC-Hsp90 was decreased by about $60 \%$ compared with the pair of BGLF4-LN/LC-Hsp90. Altogether, these results suggest the region of aa 250-295 of BGLF4 is crucial in the BGLF4/Hsp90 interaction.

The mutation of Phe-254, Leu-266, and Leu-267 of BGLF4 can disrupt the BGLF4/Hsp90 interaction

To test whether residues within the aa 250-295 region of BGLF4 are employed in the BGLF4/Hsp90 interaction, alanine substitutions were introduced. Six mutants with point mutations, Q250A, F254A, L266A, L267A, R274A, 
(a)

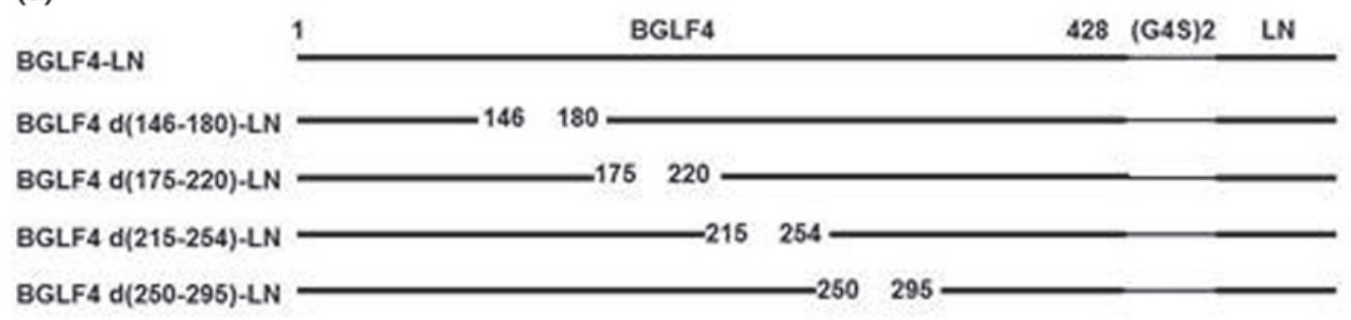

(b)

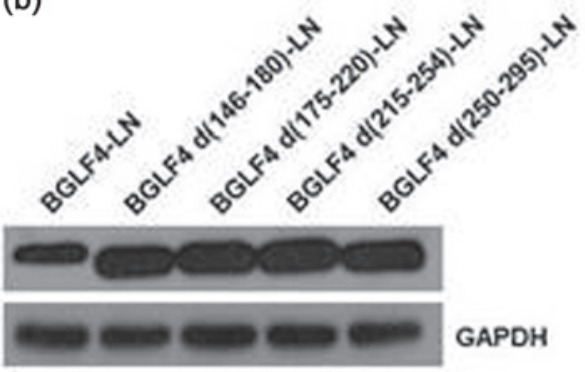

(c)

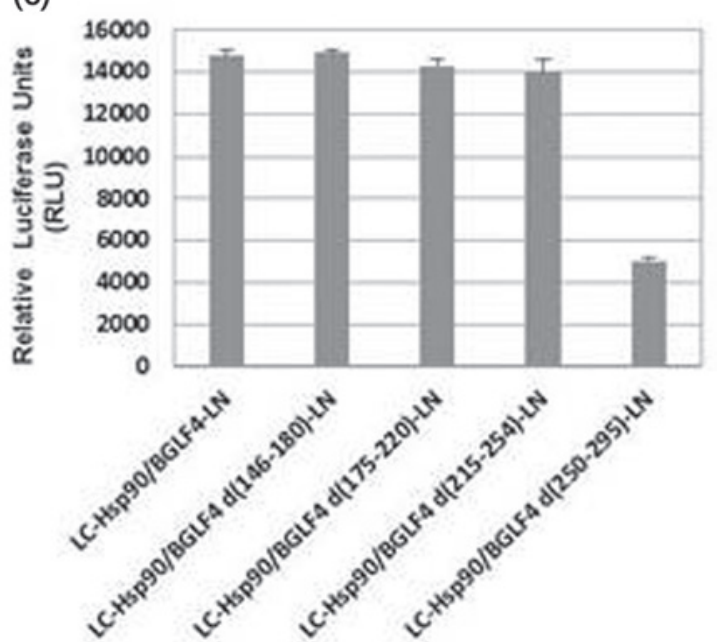

Fig. 4

Luciferase assay for the aa 150-293 region of BLGF4

(a) Deletion mutants $\mathrm{d}(146-180), \mathrm{d}(175-220), \mathrm{d}(215-254)$, and $\mathrm{d}(250-295)$ of BGLF4 tagged by HA and linked to the LN through the linker (G4S)2. (b) Western blot analysis of 293T cells transfected with deletion mutants BGLF4 d(146-180)-LN, BGLF4 d(175-220)-LN, BGLF4 d(215-254)-LN, and BGLF4 $\mathrm{d}(250-295)-\mathrm{LN}$, using the antibody against HA. (c) Luciferase assay of 293T cells co-transfected with BGLF4 d(146-180)-LN, BGLF4 d(175-220)-LN, BGLF4 d(215-254)-LN, and BGLF4 d(250-295)-LN and LC-Hsp90, after $48 \mathrm{hr}$. Data are presented as mean \pm S.D. $(\mathrm{n}=6)$.

and L266A/L267A of BGLF4 tagged by HA and linked to the LN fragment of Renilla luciferase via the linker (G4S)2, were generated (Fig. 5a). As demonstrated in Fig. 5b, western blot analysis has shown that the point mutation mutants' expression could be detected in transfected cells. To test the luciferase activity between point mutation mutants and LC-Hsp90, mutants were co-transfected with LC-Hsp90 into $293 \mathrm{~T}$ cells. After $48 \mathrm{hr}$, cells were harvested to detect the luciferase activity. As shown in Fig. $5 \mathrm{c}$, the data show that the corresponding pairs of BGLF4 (F254A)-LN/LC-Hsp90, BGLF4 (L266A)-LN/LC-Hsp90, BGLF4 (L267A)-LN/LC-Hsp90, and BGLF4 (L266A/ L267A)-LN/LC-Hsp90 restored 40-55\% luciferase activity compared with the pair of BGLF4-LN/LC-Hsp90. However, the luciferase activity of the pairs of BGLF4 (Q250A)-LN/ LC-Hsp90 and BGLF4 (F274A)-LN/LC-Hsp90 did not change obviously. These results indicate that the mutation of Phe-254, Leu-266, and Leu-267 of BGLF4 can disrupt the BGLF4/Hsp90 interaction.

\section{Discussion}

As a member of the conserved herpesviral protein kinases, BGLF4 is important for viral infection and replication (Gershburg et al., 2007; Meng et al., 2010). BGLF4 is a competent protein kinase that can phosphorylate and regulate multiple viral and cellular factors. The kinase function of BGLF4 is necessary for the optimal expression of late genes. Therapeutics targeting BGLF4 protein kinase provides a novel strategy of EBV induced diseases. The mechanism by which BGLF4 targets the nucleus has been extensively studied (Chang et al., 2012; Gershburg et al., 2010), and the crucial region of BGLF4 for its distribution was also researched. However, the interaction of Hsp90 that facilitates protein folding, stability, and intracellular trafficking, with the interacting protein was not explicit. We have previously demonstrated that BGLF4 forms a complex with Hsp90 in cells. However, the region of BGLF4 that is important for the BGLF4/Hsp90 interaction was not studied before. In this study, our findings shown that 
(a)

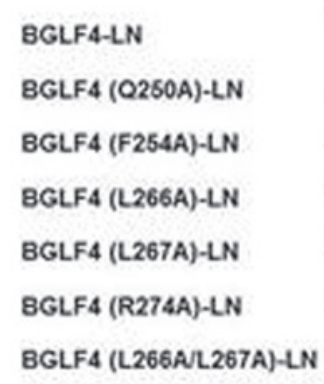

(b)

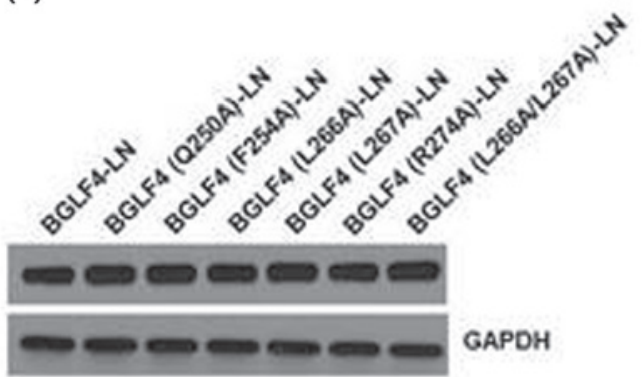

BGLF4

$428(\mathrm{G} 4 \mathrm{~S}) 2 \quad \mathrm{LN}$

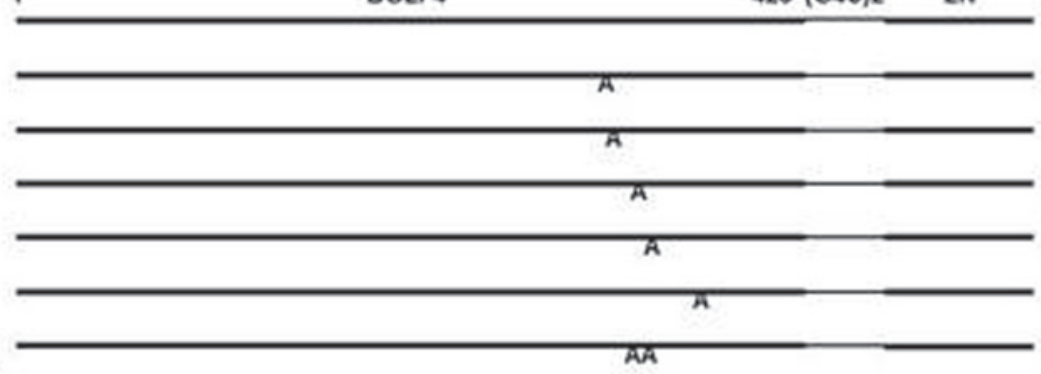

(c)

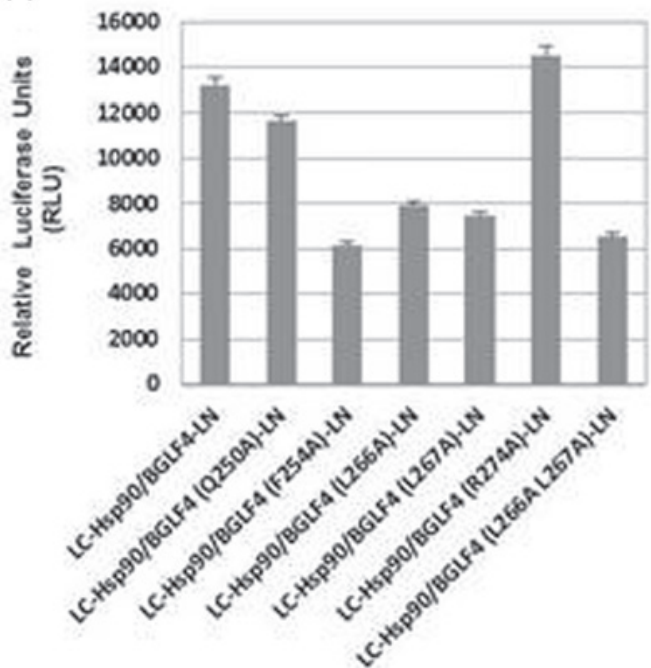

Fig. 5

Luciferase assay for BLGF4 point mutation mutants

(a) The point mutation mutants Q250A, F254A, L266A, L267A, R274A, and L266A/L267A of BGLF4 tagged by HA and linked to the LN fragment via the linker (G4S)2. (b) Western blot analysis of 293T cells transfected with point mutants BGLF4 (Q250A)-LN, BGLF4 (F254A)-LN, BGLF4 (L266A)-LN, BGLF4 (L267A)-LN, BGLF4 (R274A)-LN, and BGLF4 (L266A/L267A)-LN using antibody against HA. (c) Luciferase assay of 293T cells co-transfected with point mutants of BGLF4-LN and LC-Hsp90 after $48 \mathrm{hr}$. Data are presented as mean \pm S.D. $(n=6)$.

the SRLCA can specifically identify the interaction between BGLF4 and Hsp90.

The SRLCA is developed to determine protein-protein interactions as a bioluminescence assay and applied in preclinical cancer studies (Lake and Aboagye, 2014; Paulmurugan and Gambhir, 2003). The assay is based on the principle that a complementation of two non-functional halves of Renilla luciferase is driven by two interacting proteins. Compared with co-immunoprecipitation, the SRLCA has advantages of being simple and dynamic in living cells. The C-terminal part of BGLF4 potentially determines nuclear location (Chang et al., 2012), while the N-terminal region may modulate nuclear transportation ( $\mathrm{Li}$ et al., 2012). It is possible that the middle region of BGLF4 interacts with Hsp90. Consistent with the supposition; we have found that the aa 150-293 of BGLF4 interacts with Hsp90. In order to find which specific region of BGLF4 interacts with Hsp90, BGLF4 d(146-180)-LN, BGLF4 d(175-220)-LN, BGLF4 d(215-254)-LN, and BGLF4 d(250-295)-LN recombinants were constructed. Interestingly, our findings show that the deletion mutant BGLF4 d(250-295) obviously disrupts the BGLF4/Hsp90 interaction. It suggests that the aa 250-295 region of BGLF4 is crucial for the interaction between BGLF4 and Hsp90. Furthermore, we found that the point mutations of Phe-254, Leu-266, and Leu-267 of BGLF4 can disrupt the BGLF4/Hsp90 interaction as well.

In conclusion, the simple and specific split Renilla luciferase complementation assay can detect the interaction of BGLF4/Hsp90. It is demonstrated here that the aa 250-295 of BGLF4 interact with Hsp90. The point mutations of Phe-254, Leu-266, and Leu-267 of BGLF4 can disrupt the interaction of BGLF4/Hsp90. These results provide a rationale to develop inhibitors for disruption of the BGLF4/Hsp90 interaction. 
Acknowledgements. We thank professor Feng Li from Department of Veterinary and Biomedical Sciences, South Dakota State University for providing the valuable plasmids. This work was supported by the initiative research program of Wuhan University (No. 410100020), the advanced talent independent research program of Wuhan University (No. 410100011), and the National Natural Science Foundation of China (No. 210700228).

\section{References}

Asai R, Kato A, Kato K, Kanamori-Koyama M, Sugimoto K, Sairenji T, Nishiyama Y, Kawaguchi Y (2006): Epstein-Barr virus protein kinase BGLF4 is a virion tegument protein that dissociates from virions in a phosphorylation-dependent process and phosphorylates the viral immediate-early protein BZLF1. J. Virol. 80, 5125-5134. http://dx.doi. org/10.1128/JVI.02674-05

Chang CW, Lee CP, Huang YH, Yang PW, Wang JT, Chen MR (2012): Epstein-Barr virus protein kinase BGLF4 targets the nucleus through interaction with nucleoporins. J. Virol. 86, 8072-8085. http://dx.doi.org/10.1128/JVI.01058-12

Chang CW, Lee CP, Su MT, Tsai CH, Chen MR (2015): BGLF4 kinase modulates the structure and transport preference of the nuclear pore complex to facilitate nuclear import of Epstein-Barr virus lytic proteins. J. Virol. 89, 1703-1718. http://dx.doi.org/10.1128/JVI.02880-14

Chee MS, Lawrence GL, Barrell BG (1989): Alpha-, beta- and gammaherpesviruses encode a putative phosphotransferase. J. Gen. Virol. 70 ( Pt 5), 1151-1160. http://dx.doi. org/10.1099/0022-1317-70-5-1151

Gershburg E, Raffa S, Torrisi MR, Pagano JS (2007): Epstein-Barr virus-encoded protein kinase (BGLF4) is involved in production of infectious virus. J. Virol. 81, 5407-5412. http://dx.doi.org/10.1128/JVI.02398-06

Gershburg S, Murphy L, Marschall M, Gershburg E (2010): Key motifs in EBV (Epstein-Barr virus)-encoded protein kinase for phosphorylation activity and nuclear localization. Biochem. J. 431, 227-235. http://dx.doi.org/10.1042/ BJ20100558

Hattori M, Ozawa T (2014): Split luciferase complementation for analysis of intracellular signaling. Anal. Sci. 30, 539-544. http://dx.doi.org/10.2116/analsci.30.539

Iwahori S, Murata T, Kudoh A, Sato Y, Nakayama S, Isomura H, Kanda T, Tsurumi T (2009): Phosphorylation of p27Kip1 by Epstein-Barr virus protein kinase induces its degradation through SCFSkp2 ubiquitin ligase actions during viral lytic replication. J. Biol. Chem. 284, 18923-18931. http://dx.doi.org/10.1074/jbc.M109.015123

Jego G, Hazoume A, Seigneuric R, Garrido C (2013): Targeting heat shock proteins in cancer. Cancer Lett. 332, 275-285. http://dx.doi.org/10.1016/j.canlet.2010.10.014

Lake MC, Aboagye EO (2014): Luciferase fragment complementation imaging in preclinical cancer studies. Oncoscience 1 , 310-325. http://dx.doi.org/10.18632/oncoscience. 45

Lee CP, Huang YH, Lin SF, Chang Y, Chang YH, Takada K, Chen MR (2008): Epstein-Barr virus BGLF4 kinase induces disassembly of the nuclear lamina to facilitate virion production. J. Virol. 82, 11913-11926. http://dx.doi. org/10.1128/JVI.01100-08

Li R, Wang L, Liao G, Guzzo CM, Matunis MJ, Zhu H, Hayward SD (2012): SUMO binding by the Epstein-Barr virus protein kinase BGLF4 is crucial for BGLF4 function. J. Virol. 86, 5412-5421. http://dx.doi.org/10.1128/JVI.00314-12

Meng Q, Hagemeier SR, Kuny CV, Kalejta RF, Kenney SC (2010): Simian virus $40 \mathrm{~T} / \mathrm{t}$ antigens and lamin A/C small interfering RNA rescue the phenotype of an Epstein-Barr virus protein kinase (BGLF4) mutant. J. Virol. 84, 4524-4533. http://dx.doi.org/10.1128/JVI.02456-09

Nahleh Z, Tfayli A, Najm A, El Sayed A, Nahle Z (2012): Heat shock proteins in cancer: targeting the 'chaperones'. Future Med. Chem. 4, 927-935. http://dx.doi.org/10.4155/fmc.12.50

Paulmurugan R, Gambhir SS (2003): Monitoring protein-protein interactions using split synthetic renilla luciferase proteinfragment-assisted complementation. Anal. Chem. 75, 1584-1589. http://dx.doi.org/10.1021/ac020731c

Pearl LH, Prodromou C (2000): Structure and in vivo function of Hsp90. Curr. Opin. Struct. Biol. 10, 46-51. http://dx.doi. org/10.1016/S0959-440X(99)00047-0

Rossi F, Charlton CA, Blau HM (1997): Monitoring protein-protein interactions in intact eukaryotic cells by beta-galactosidase complementation. Proc. Natl. Acad. Sci. USA 94, 8405-8410. http://dx.doi.org/10.1073/pnas.94.16.8405

Sun X, Bristol JA, Iwahori S, Hagemeier SR, Meng Q, Barlow EA, Fingeroth JD, Tarakanova VL, Kalejta RF, Kenney SC (2013): Hsp90 inhibitor 17-DMAG decreases expression of conserved herpesvirus protein kinases and reduces virus production in Epstein-Barr virus-infected cells. J. Virol. 87, 10126-10138. http://dx.doi.org/10.1128/JVI.01671-13

Taipale M, Krykbaeva I, Koeva M, Kayatekin C, Westover KD, Karras GI, Lindquist S (2012): Quantitative analysis of HSP90-client interactions reveals principles of substrate recognition. Cell 150, 987-1001. http://dx.doi. org/10.1016/j.cell.2012.06.047

Wang JT, Doong SL, Teng SC, Lee CP, Tsai CH, Chen MR (2009): Epstein-Barr virus BGLF4 kinase suppresses the interferon regulatory factor 3 signaling pathway. J. Virol. 83, 1856-1869. http://dx.doi.org/10.1128/JVI.01099-08

Wang JT, Yang PW, Lee CP, Han CH, Tsai CH, Chen MR (2005): Detection of Epstein-Barr virus BGLF4 protein kinase in virus replication compartments and virus particles. J. Gen. Virol. 86, 3215-3225. http://dx.doi.org/10.1099/vir.0.81313-0

Whitesell L, Lindquist SL (2005): HSP90 and the chaperoning of cancer. Nat. Rev. Cancer 5, 761-772. http://dx.doi. org/10.1038/nrc1716

Yang PW, Chang SS, Tsai CH, Chao YH, Chen MR (2008): Effect of phosphorylation on the transactivation activity of Epstein-Barr virus BMRF1, a major target of the viral BGLF4 kinase. J. Gen. Virol. 89, 884-895. http://dx.doi. org/10.1099/vir.0.83546-0

Zhu J, Liao G, Shan L, Zhang J, Chen MR, Hayward GS, Hayward SD, Desai P, Zhu, H (2009): Protein array identification of substrates of the Epstein-Barr virus protein kinase BGLF4. J. Virol. 83, 5219-5231. http://dx.doi.org/10.1128/ JVI.02378-08 